\title{
Die historische Litteratur des Niederrheins für das Jahr 1899. Yon
}

\section{Kaspar Keller.}

\section{A. Römische Zeit.}

1. Museographie.

a) H. Nissen. Bericht über die Thätigkeit des Nationalmuseums in Bonn vom 1. April 1898 bis 31. März 1899. - Nachrichten über Deutsche Alterthumsfunde 1899, s. $33-36$.

b) H. Nissen. Bonn. Provinzialmuseum. WZ. 18, S. 416 bis 419 .

c) Poppelreuter. Köln. Museum Wallraf-Richartz. - WZ. 18 , S. $419-420$.

d) J. Hansen. Köln. Historisches Museum. WZ. 18, S. 420.

e) A. Kisa. Aachen. Städtisebes Suermondt-Museum. - WZ. 18, s. $420-421$.

f) 0. Schell. Elberfeld. Sammlungen des Bergischen Geschichtsvereins. - WZ. 18 , S. 421.

g) J. Steiner. Xanten. Niederrbeinischer Alterthumsverein. - WZ. 18, S. 421.

2. Y. Rheinische Kultur zur Römerzeit. - Köln. Volksztg. 1899. nr. 437 .

3. H. Nissen. Caesars Rheinfestung. A. Zur Geschichte. Mit 1 Textfigur. JVARb. 104, S. 1-29.

4. C: K o e ne n. Caesars Rheinfestung. B. Die Ausgrabungen im Winter 1898/99. Mit 9 Tafeln. - JVARh. 104, S. 30 bis 55 .

5. Wolf. Wo bante Caesar seine Rheinbrïcke? Köln. Tagebl. 1899, nr. 61. 
Die historische Litteratur des Niederrheins für das Jahr 1899. 143

6. Wulff. Julius Caesars Rheinbriicke. RheinGBll. 4, S. 260 bis 270 .

Nissen und Koener weisen nach, dass Caesars Rheinübergänge in Neuwieder Becken stattgefunden haben und dass die von Caesar zur Deckung seiner zweiten Rheinbrücke angelegte Befestigung zwischen Urmitz und Weissenthurm in der Nähe des Urmitzer Bahnhofs gelegen habe. Wolt und Wulff halten an der Gegend zwischen Köln und Bonn als Uebergangsstelle fest.

7. A. K is a. Die antiken Gläser der Frau Maria vom Rath, geb. Stein zu Köln. Mit 33 Tafeln. Bonn, Georgi 4․ $199 \mathrm{~S}$.

Es ist noch nicht allzulange her, dass man die Existenz rheinischer Glaswerkstätten zur Römerzeit zugiebt, veranlasst durch die Autdeckung von römischen Glasfabriken und Glasöfen zu Cordel bei Trier, bei der Saalburg und zu Köln. Kisa giebt einen aus vertrauter Sachkenntniss hervorgegangenen Ueberblick über Geschichte und Technil: der antiken Glasindustrie und weist dem rheinischen Zweige des Glaskunstgewerbes seine gebührende Stelle an. Der grosse Umfang desselben, sowie seine Eigenart und allmäliche Loslösung von ausländischen Mustern wird nachgewiesen. Etwa von Mitte des II. Jahrhunderts ab übernahmen die gallisch-rheinıschen Glashütten neben Alexandrien die Führung und Weiterbildung dieser Industrie. Auf 33 grösstentheils farbigen Tateln werden die meisten Stücke der Sammlung, fast durchweg Kölner Funde von vorzüglicher Erhaltung, abgebildet.

8. H. Lehner. Effern bei Köln. Römische Grabkammer. Mit 6 Abbildungen. JVARh. 104, S. 168-173.

Dieses Grab bildet ein Seitenstiuck zu dem bekannten Römergrab bei Weiden. Es enthält zwei Sarkophage, deren Inhalt aber früher geraubt worden ist, wobei auch wohl die Deckel zerschlagen wurden.

9. H. Lehner. Klein Boslar. Römisches Begräbniss. JVARh. 104 , S. $173-174$.

10. H. Boschheidgen. Asciburgiun. Ein Ausgrabungs- und Beobachtungsbericht. Mit 1 Tafel und 23 Textfiguren. JVARh. 104 , S. $136-163$.

Ausführlicher Bericht über frühere und eigene Ausgrabungen. $B$ hat die Lage und den Umfang des römisehen Kastells nachgewiesen, (1) hat ferner nachgewiesen, dass bei Asciburgium ein Hafen bestanden habe und dass unter dem im Itinerarium Antoninum zwischen Vetera und Novesia rorkommenden Calo unser Asciburgium zu verstehen sei. Im Anhang wird ein Verzeichniss der von B. gemachten Einzelfunde gegeben.

11. W. Bösken. Altertbumsfunde bei Alpen (Kreis Mörs). Mit 2 Textfiguren. JVARh. 104, s. 117-126.

Verf. weist $u$. a. nach, dass in Alpen nur eine Burg gestanden 
habe, während Clemen (Kunstdenkmäler des Kreises Mörs) noch zwei Burgen, eine obere und eine untere annahm.

12. H. Lehner. Juppiter mit dem Giganten. Mit 1 Texttigur. JVARh. 104, S. $62-64$.

Den von Knickenberg (vgl. Litteraturber. 1892, nr. 28) beschriebenen Votirstein unterzieht $L$. einer erneuten Untersuchung; er weist nach, dass auf dem Stein Juppiter mit dem Giganten, mit der rechten Hand den Blitz schleudernd, dargestellt sei.

13. G. Il estwerdt. Sigillata-Stempel und -Formen der städtischen Alterthumssammlung in Kleve. - JVARh. 101, S. 112-116.

\section{B. Mittelalter und Neuzeit.}

\section{Quellen und Quellenkunde.}

14. J. Se e müller. Zum Annolied. Sendschreiben an Edwin Schröder. - Zeitschrift für Deutsches Alterthum. 42, S. 322-338.

Schroder hatte in seiner Ausgabe der Kaiserchronik die Ansicht ausgenprochen, dass das Annolied unmittelbar von dem Verfasser der Kaiserchronik beoutzt worden sei. Demgegenüber tritt Seemüller für die von Roediger in seiller Ausgabe des Annoliedes aufgestellte Behauptung ein, dass das Annolied und die Kaiserchronik unabhangig von einander eine gemeinsane Quelle, eine verlorene deutsche Chronik benutzt hatten.

15. St. Ehses. Nuntiaturberichte aus Deutschland nebst ergänzenden Aktenstucken, 1585 (1584)-1590. Erste Abtheilung: Die Kölner Nuntiatur. Zweite Hälfte. Ottavio Mirto Frangipani in Köln 1587-1590. [A. u. d. T. Quellen und Forschungen auf dem Gebiete der Geschichte. In Verbindung mit ibrem historiscben Institut in Rom herausgegeben vou der Görresgesellschaft. VII. Band.] Paderborn, Schöningh. LXI, j43 S.

An der Sammlung des Materials für diesen Band ist noch A. M e is te $r$ betheiligt. Die Bearbeitung hat Ehses besorgt. Die Einleitung bringt zunächst den Lebenslauf des Nuntius Frangipani, der ein würdiger Nachfolger Bonomis war, und dann die Schilderung semer Thàtigkeit auf der Kölner Nuntiatur. Hier handelte es sich vor Allem um die Regelung der Verháltnisse im Erzstift Köln. Frangipanis Aufgabe war eine doppelte; auf kirchlichem Gebiete galt es die Durchführung der Reformdekrete des Concils von Trient, auf weltlichem die Ordnung der finanziellen Verhältnisse des Erzstifts. Diese war um so 
Die historische Litteratur des Niederrheins für das Jahr 1899. 145

(hwleriger, als grosse Theile des Stiftes von ren Hollandern und von den Freibeuter Martin Schenck ron Nideg.gen besetzt waren. Nachdem $\therefore$ mıt Hülte der Spanier gelungen war, das Gebiet zu säubern, schloss $\therefore$ mit den alten und neuen Schuldnern Vergleiche ab, die eine allm.lhliche Besserurg herbeizuführen versprachen. Das grösste Hinderniss : iir die Reformen auf beiden Gebieten bildete der Erzbischof Ernst ron Baiern selber. Eine zweite Hauptaufoabe des Nuntius bestand in der itcherung des Katholizismus in den Landen des Herzogs von JülichCleve-Berg und in der Zurücktïhrung des katholischen Rathes nach tuchen. Annähernd 400 Aktenstücke werden, fast sammtluch dem vollen Wortlaute nach, zum Abdruck gebracht.

16. A. Meister. Die Finalrelation des Kölner Nuntius Johann Baptista Bussi. - Römische Quartalschrift. 13, S. 347--364.

J) Ne Nuntiatur Bussis fällt in die Jahre 1706-1712. Die Nuntiatur gewann damals an Bedeutung, weil der Kurfïrst Joseph Clemens wegen -eines Anschlusses an Ludwig XIV. nach Frankreich hatte flüchten munsen Grosse Entschiedenheit zeigte Bussi bei dem Versuche des b..udenburgischen Residenten in KöIn, den Calvinismus in diese Stadt emzuführen. Die Finaluelation Bussis ist schon als solche interessant, dein en sind uns nur wenige ủber Kölner Nuntiaturen erhalten.

17. Ed. Simans. Die Mechterner Predigten nach Teschenmachers ungedruckten Kirchenannaleu. - Theologische Arbeiten aus dem Rhein. wissensch. Prediger-Verein. Nene Folge. 3, S. 70 bis 83 .

Graf Adolf von Neuenahr liess im Juli 1582 vor den Thoren der Stalt Koln eine protestantische Predigt halten. Diese Predigt ist im Druck erschienen, doch war Text und Titel davon unbekannt. Simons welst nach, dass eine Abschrift der Predigt in Teschenmachers Annalen rntlalten ist, und dass der Prediger Johannes Christiani aus Otzenrath, ter Mitbegründer der niederrheinischen Synodalverfassung, war. Die Nachrucht, dass die Predigt im náchsten Jahre, 1583, wiederholt worden rel, wird als unrichtig erwiesen.

18. Nebe. Drei Briefe über Peter Lo's Verhandlungen mit den Wiedertäufern in Blankenberg, 1565. - ZBergGV. 34, S. 1 bis 15 .

Drei Briefe des Düsseltlorfer Prediger's Peregrinas Wilach an Geory Cassander. Dieser war zuerst bestimmt, mit den auf der Burg a B Blankenberg festgesetzten Wiedertiufern zu verhandeln, wurde aber durch Krankheit daran gehindert.

19. W. Harless. Zeugenaussagen betreffend die reformirten Gemeinden der Klassen Solingen und Elberfeld vor und nach 1609. - ZBergGV. 34, S. 17-64.

Die Aussagen sind im Jahre 1648 durch einen Notar aufgenommen. Nan erkennt daraus die Zeiten der ersten Bildung der Gemeinden und 
die Einwirkungen der Gegenreformation, und wie aut eine Zeit der stillen ungestörten Entwicklung der Rückschlag in den Jahren 16251629 folgte.

20. Aus einem Aktenstïcke, betreffend die öffentliche Religionsiibung reformirter Gemeinden im Bergischen, 1624. - ZBergGV. 34, S. 16 .

21. B. Schirrmacher. Drei unbekannte Streitschriften aus der Zeit des Jülich-Clevischen Erbfolgekrieges, mit einem Anhange: Zwei neue Handschriften des „Strahlendorff'schen Gutachtens". - Rostock, Selbstverlag. Rostocker Dissertation. $127 \mathrm{~S}$.

Der Erbfolgestreit um die Lande Jülich-Cleve-Berg hat wegen der verwickelten Frag'e der Erbberechtigung der Prätendenten eine reiche Litteratul hervorgerufen. Drei angeblich bisher unbekannte Streitschriften hat Schirtmacher in einem handschriftlichen Sammelbande der Rostocker Universitätsbibliothek aufgefunden und giebt eine modern deutsche Uebersetzung davon. In zahlreichen Anmerkungen wird versucht, den historischen Werth der Schriften einer kritischen Untersuchung zu unterziehen. Aus den zwei auf der Rostocker Universitätsbibliothek aufgefundenen Handschriften des Strahlendorfi"schen Gutachtens werden die Varianten von dem Droysenschen Druck mitgetheilt.

22. H. Sehafstaedt. Köln und Kurprinz Georg Wilheln. ZBergGV. 34, S. 93-95̃.

Bericht über die Fahrt des Kurprinzen an Köln vorbei, aut der Rückfahrt von seiner Verınählung in Heidelberg.

23. G. C. Knod. Rheinländische Studenten im XVI. und XVII. Jahrhundert auf der Universität Padua. - AnnHVNiederrh. 68 , S. $133-189$.

24. A. 'Tille. Uebersicht über den Inhalt der kleineren Archive der Rheinprovinz. - AnnHVNiederrh. Beiheft IV. Enthält die Kreise: Mülheim a. Rh.-Stadt und -Land; Wipperfürth; Gummersbach; Waldbröl und Sieg.

Die vier Beihefte sind auch unter besonderem Titel als Erganzungsband I zu den AnnHVNiederrh. erschienen.

25. H. Diemal. Hessen und die Reichsstadt Köln im 15. Jahrhundert. Regesten zur Hessischen und Deutschen Geschichte. Giessen, von Münchow. $186 \mathrm{~S}$. Sonderabdruck aus: Mittheilungen des Oberhessischen Geschichtsvereins. N. F. VII.

26. Fr. Lau. Das Buch Weinsberg. Kölner Denkwürdigkeiten aus dem XVI. Jahrhundert. Vierter Band. Mit dem Köher Stadtplan vom Jahre 1571. [A. u. d. T. Publikationen der 
Die historische Litteratur des Niederrheins für das Jahr 1899. 147

Gesellschaft für Rheinische Geschichtsknnde XVI.] Bonn, Hanstein XXII, 323 S.

Dieser Band bringt den Schluss der Aufzeichnungen Weinsbergs ron 1588-1598. Ueber Todesjahr und Todesart des Verfassers erfahren wir Nichts Der Band enthïlt ein von Blumschein bearbeitetes Glossar und das Register zum 3. und 4. Band.

27. J. Dreesen. Kölner Knltur im XVI. Jahrbundert. Die Handschrift des Hermann von Weinsberg. Mittheilungen und Erläuterungen. - Köln, Schmitz. VI, $145 \mathrm{~S}$.

Bringt kulturgeschichtlich interessante Auszüge.

28. H. Isay. Exemplar der Ausgabe der Kölner Statuten rom Jahre 1437 in der Stadtbibliothek zu Trier. - Trierer Archiv. I. S. 99.

29. H. Keussen. Inhaltsverzeichniss zu der Sammlung der Rathsedikte. $1493-1819$ (43). - MStadtAKöln. 29, S. 159-336. Das Verzeichniss ist angefertigt von $H$. Nottbrock.

30. A. Tille. Theuerung in Köln. KBWZ. 18, S. 40-41.

31. P. Loë. Das Kalendarium der Universität zu Köln. AnnHVNiederrh. 67, S. $109-129$.

32. B. Duhr. Die ältesten Studienpläne des Jesuitengymnasiums in Köln. - Mittheil. der Gesellschaft für Deutsche Erz.- und Schul-Geschichte. 8, S. $130-146$.

33. J. Petry. Die Hausordnung der Fraterherren und der Tabernakelstiftung zu Emmerich. Ein Beitrag zur Geschichte der Internatserziehung. - Jalıresbericht des Progymnasiums zu Steele. S. 1-19. Steele, Druck von Berthold $4^{\circ}$.

Die Hausordnung der Fraterherren ist nach dem Einzug der Jesuiten in das Emmericher Gymnasium. c. 1592, erlassen worden, die der Tabernakelstiftung noch später. Jem lateinischen Text ist eine deutsche Uebersetzung beigegeben.

34. B. Liesen. Die älteste bisher ungedruckte Schul- und Studienordnung des Emmericher Gymnasims. - Beilage zum Programm des Gymnasiums zu Enmerich. Emmerich, Romen. $4^{\circ}$. XIII, $8 \mathrm{~S}$.

Die Schulordnung ist zwischen 1566 und 1572 vom Dechant Hermann Schilder nach einer ältern Schulordnung abgefasst worden, giebt also ein Bild des Gymnasiums in der ersten Hälfte des XVI. Jahrhunderts. 35. C. vom Berg. Auszug aus der Matrikel der Hohen Schule zil Herborn. - Monatsschr. BergGV. 6, S. 29-34.

Verzeichnet die Bergischen und Jülichschen Studenten von 1586 an. 
36. H. Veltmann. Aachener Prozesse am Reichskammergericht. II. Abtheilnng. Prozesse aus dachen und dem Regierungsbezirk Aachen mit Ausnahme der die Aachener Behörden und Korporationen betreffenden. Schluss. - ZAachenGV. 21, S. 1 bis 59 .

Vgl. Litteraturbericlit 1896, nr. 111.

37. A. Bellesbeim. Beiträge zur Geschichte Aachens im 16. Jahrhundert. - ZAachenGV. 21, S. 122--134.

Die auf Aachen bezüglichen Urkunden aus den von Sehwarz herausgegebenen Nuntiaturberichten Kaspar Groppers (vgl. Litteraturbericht 1898, nr. 28] und den von Ehses heraungegebenen Nuntiaturberichten Frangipanis [vgl. oben nr. 15].

38. W. Brüning. Eine Aachener Chronik 1770-1796. - Aachen, Cremer. 54 S. Sonderabdruck aus MVAachenVorzeit, Bd. 11.

39. F. X. Bosbach. Neues Material zur Geschichte Burtscheids. - ZAachenGV. 21, S. 262-263.

Fund einer Kiste mit Archivalien.

40. Urkunde betreffend Verkauf eines Gutes zu Itterbach an die Abtei Heisterbach. - ZBergGV. 34, S. 148.

41. W. Sauer. Zur Geschichte der Besitzungen der Abtei Werden. Fortsetzung. II. Die Güter der Abtei im vormaligen Hochstift Münster. - ZBergGV. 34, S. 210 --266.

42. W. Br a chm iiller. Zur Wirthschaftsgeschichte eines rheinischen Klosters im 15. Jahrhundert. Nach einem Rechnungsbuche des Klosters Walberberg ans dem Jahre 1415. - WZ. 18 , S. $266-308$.

In der Einleitung giebt $\mathrm{Br}$. eine Darstellung über den Grundbesitz dieses Cisterciensernonnenklosters und die darauf betriebene Eigenwirthschaft, eine Zusammenstellung der Einnahmen und Ausgaben und eine Uebersicht über Löhne and Preise, die er mit gleichzeitigen Kölner Verhältnissen in Vergleich stellt.

43. Köllmann. Urkunden des Klosters Saarn aus dem 13. und 14. Jahrhundert. Fortsetzung. - MonatsschrBergGV. 6, S. 45 bis 52 .

44. W. S a u e r. Urkunden und Regesten zur Geschichte des Augustiner-Eremitenklosters Marienthal bei Brünen. Mit zwei Beilagen. - ZBergGV. 34, S. 179-209.

45. Weisthum das Gustorfer Broich betreffend. - Beitr. z. Gesch. von Neuss-Grevenbroich. 1, S. 78-80. 
Die historische Litteratur des Niederrheins für das Jahr 1899. 149

46. Urkunde betreffend Schloss und Herrschaft Arburg. 1329. ZBergGV. 34, S. 142.

47. Urkunden betreffend die Vogtei Weeze und Herrlichkeit Hertefeld. 1369, 29. April. - ZBergGV. 34, S. 96.

48. Urkunden zur Geschichte des Dönhofs und der Freiherren von Elverfeld, sowie anderer Adelsgeschlechter der Grafschaft Mark. - ZBergGV. 34, S. 133-141.

49. Bornefeld. Gerechtigkeit wegen des Mahlens der Einwohner von Lüttringhausen aus dem Jahre 1595. - Monatssebr. BergGV. 6, S. 146-153.

50. C. vom Berg. Auszige aus den Akten des Pfarrarchivs zu Remlingrade. - Monatsschr. BergGV. 64, S. 69-82.

Rechnungen aus dem Jahre 1689 u. A.

əั1. 0 . Winckelmann. Zur Geschichte Sleidans und seiner Commentare. ZeitschrGOberrhein.

52. J. Grunau. Beiträge zur Geschichte der Kreise Neuss-Grevenbroich. Beilage zur Neuss-Grevenbroicher Zeitung. - Neuss, Druck der Gesellschaftsdruckerei.

Im Folgenden citirt als: BGNeuss-Grevenbroich.

\section{Darstellende Arbeiten allgemeineren Inhalts.}

53. H. G. Schmidt. Ueber die Ernennung des Bonifatius zum Metropoliten von Köln. - Kieler Inauguraldissertation. Coe. penick, Jennes $45 \mathrm{~S}$.

Verf. beweist, dass Bonifatius thatsächlich zum Metropoliten des Kölner Stuhles ernannt, aber durch den Widerstand eines grossen Theiles der Geistlichkeit und die unzuverlässige Interessenpolitik der frankischen Fürsten an der Besitznahme des Kölner Stuhles verhindert worden ist.

54. A. Peters. Die Reichspolitik des Erzbischofs Philipp von Köln (1167-1191). - Marburger Dissertation. Marburg, Universitätsbuchdruckerej. $107 \mathrm{~S}$.

Philipp von Heinsberg war der letzte der grossen, kaiserlichgesinnten und vom Kaiser eingesetzten Kölner Erzbischöfe. Er wandelte zunachst die Spuren seines Vorgängers Reinald von Dassel im Kampfe für die staufisch-kaiserlichen Irteressen in Italien. In der zweiten Hälfte seiner Regierung änderte sich das Verhältniss zum Kaiser. Beim Ausbau seiner landesherrlichen Stellung gerieth er in einen wirthschaftlichen 
und politischen Gegensatz zum Staufischen Hause. An Philipps Opposition scheiterte die Begründung der staufischen Dynastie. Einen rorherrschenden Einfluss auf diese Schwenkung in Philipps Politık übte die Stadt Köln nit jhren wichtigen Handelsinteressen aus. Nit Philipp beginnt die Reihe der aus landesherrlichen Interessen welfisch und englisch gesinnten vom Kaiser unabhängigen Kölner Erzbischöte.

55. H. Schrohe. Die politischen Bestrebungen Errbischof Siegfrieds von Köln. Ein Beitrag zur Geschichte des Reiches unter den Königen Rudolf von Habsburg' und Adolf von Nassau. - AnnHVNiederrh. 67, S. 1-108; 68, S. 54-108.

56. C. Varrentrapp. Zur Charakteristik Herrmanns von Wied, Bucers und Groppers. - Zeitschr. für Kirchen-G. 20, S. 37 -58 .

57. A. Meister. Der Ntrassburger Kapitelstreit 1583-1592. Ein Beitrag zur Geschichte der Gegenreformation. -- Strassbura, Heitz. XV, $428 \mathrm{~S}$.

Das vortreffliche Buch Meisters ist auch für die niederrheinischen Verhältnisse insofern von Wichtiglkeit, als die protestantischen Kölner Domherrn und später auch Gebhard Truchsess selber nach ihrer Niederlage in Köln den Kampf nach Strassburg verpflanzten, und auch die Haltung der rheinischen geistlichen Karfürsten gegenüber den Strassburger Wirren in Betracht kommt.

58. A. Rosenlebuer. Die Stellung der Kurfürsten Max Emanuel von Baiern und Josef Clemens von Köln zur Kaiserwahl Karls VI. (1711). - [A. u. d. T. Heigel-Grauert, Historische Abhandlungen. Heft 13.] München, Lüneburg. $148 \mathrm{~S}$.

59. L. Schmedding. De regeering van Frederik van Blankenheim, busschop van Utrecht. Leiden, van Leeuwen. XIII, $286 \mathrm{~S}$.

Friedrich regierte von $1393-1423$.

60. K. R e m b e rt. Die ,Wiedertäufer" im Herzogthum Jülich. Studien zur Geschichte der Reformation, besonders am Niederrhein. - Berlin, Gaertner. XI, 637 s.

Rembert will „für seinen Theil endlich denen nach Gebühr Gerechtigkeit widerfahren lassen, die jahrhundertelang verfolgt sind, und einen Theil der Schuld abtragen, die durch ihr meist unschuldig geflossenes Blut angehäuft ist". Mit Keller hält er an dem ununterbrochenen Zusammenhang der "Taufgesinnten“, wie sich die Wiedertäufer meist selber nannten, mit den mittelalterlichen Ket\%ern fest, deren Vorkommen besonders zahlreich für den Niederrhein bezeugt ist: anders sei das Auftauchen zahlreicher Täufergemeinden so kurz nach dem Auftreten 
Die historische Litteratur des Niederrheins für das Jahr 1899. 151

Juthers nicht zu erklären. Vorgearbeitet sei der Bewegung auch durch die zahlreichen Niederlassungen der Brüder rom gemeinsamen Leben. Ils wissenschaftliche Vorarbeit benutzten die Taufer vor Allem die Schriften des Erasmus von Rotterdam. Die Täuferbewegung tand puldung und Fückhalt bei einer Anzahl von Jülichschen Adligen. Unter diesen ragte als besonderer Gönner Werner von Palant, Drost zu Wassenberg hervor. Hier, im nordwestlichen Winkel des Herzogthums Jülich strömten von allen Seiten die zusammen. die von andern Orten hatten tliehen müssen. So fanden sich auch zahlreiche Prediger ein, die man yon diesem ihrem Aufenthaltsort auch kurz die Wassenberger Prädikanten nennt, so Campanus, Klopreis, Vinne. Slachtseap, Roll u. A. Diese gingen zwar ursprünglich von verschiedenen religiösen Richtungen aus, traten aber schliesslich in Münster offen zur Wiedertaufe über. Der Lebenslauf der einzelnen Prädikanten, von denen eine Anzahl dem Herzogthum Jülich selbst entstammten, wird zum Theil in breitester Wrise (Campanus) geschildert. Im weiteren werden die Verbindungen der Táufer im Herzogthum Jülich mit Münster dargelegt und die Verfolgungen geschildert, denen die Täufer nach der Einnahme Münsters auscresetzt waren. In späterer Zeit tanden die Täufer Zuflucht in d‘r Herrlichkeit Rheydt und in der Grafschaft Mörs, besonders in Crefeld. Diese Stadt verdankt ihre Blüthe nicht zum geringsten Theil gerade den dorthin geflüchteten Täufern (Mennoniten). Im 18. Jahrhundert trat dann eine allgemeine Duldung dieser religiösen Bewegung ein.

61. F. Schroeder. Aus der Zeit des klevischen Erbfolgestreites.

- Historisches Jahrbuch der Görresgesellschaft. 19, S. 305

bis 335 und $792-826$. 20, S. 25-54;213-235 und 773 bis 805 (Anhang).

In sehr ausführlicher Weise schildert Schröder die Verfolg ungen, demen die Katholiken in Goch im Jahre 1615 und in den folgenden Jahren ausgesetzt waren. Den Anlass zu diesen Verfolgungen gab ein angeblich von den Katholiken Gochs gewen den Erbprinzen Georg Wilhelm von Brandenburg aus Anlass seiner Anwesenheit in Goch geplantes Attentat. Die Haltlosigkeit der Anschuldigung wurde durch die Untersuchung bald dargethan. Interessant ist die Darstellung, wie der Urheber dieser falschen Denunciation, der protestantische Prediger Ceporinus in Goch, durch die brandenburgischen Behörden mit allen Mitteln, Gewalt und Rechtsbeugung, der verdienten Strafe entzogen wurde. An den Folgen dieser ungerechten Anklage hatten die Katholaken Gochs noch bis ins 18. Jahrhundert hinein zu leiden. Genaue Nachrichten über den Verlauf dieser Angelegenheit erhalten wir durch dav niederdeutsche Gedicht der Fran Petronella van Heuckelum, der Tochter des Bürgermeisters Goswin Eyken, der das Haupt der Ver. chwörung gewesen sein soll, und Ehefiau des mitangeklagten Dietrich van Heuckelum. Im Anhang wird eine Anzahl in Betracht kommender Aktenstücke abgedruckt. 
62. Von Werthern. Fürstliche Besuche in Wesel. Ein Rückblick auf fünf Jahrluunderte. - Wesel, Fincke und Mallinckrodt. 1. Heft, 1898, S. $1-54$. 2. Heft, S. $55-149$, und XXIV S. 3. Heft, S. $150-268$, and XXXIV $\$$.

Eine sehr fleissige Zusammenstellung aller fürstlichen Busuche in Wesel, wobei auch dic in Betracht kommenden Zeitereignisse dargestellt werden, allerdings von einseitig-preussischem Standpunkte aus.

63. O. Sichell. Historische Wanderungen durchs Bergische Land. III. Die Dhün und ilı Gebiet. - MonatsschrBergGV. 6, S. $25-29 ; 59-64 ; 93-96 ; 141-144 ; 167-171 ; 216$ bis 243.

64. J. Leithaeuser. Ortsnamen im Wuppergebiete. - ZBergGV. 34, S. $97-122$.

$\mathrm{L}$ unternimmt eine etvmologische Untersuchung der Ortsnamen im Wuppergebiete und in den angrenzenden Gebieten Rheinlands und Westfalens. Um dio grosse Anzahl der Namen beherrschen zu können, gruppiert er sie nach den Grundbegriffen und bildet so Abschnitte wie: Wasser, Berg, Wald, Rodung, Kirche u. a. Im vorliegenden ersten Theil werden die Ortsnamen mit dem Begriff Wasser im weitesten Sinne behandelt.

65. Protze. Die Münzstätten des Bergischen Landes. - MonatsschrBergGV. 6, S. $1-4$.

Die älteste Münzstätte war Wielberg. Im Jahre 1275 wurde di’ Münze mit Ënwilligung König Rudolfs nach Wipperfürth verlegt.

66. W. Brüning. Deutsche Münzwerthe vom Jahre 1656 bis zur Fremdberrschaft. - MVAachenVorzeit, 12, S. 45-48.

67. J. Leith a euser. Ältere Münznamen. - MonatsschrBergGV. 6, S. $14-16$.

68. C. Rademacher. Germanische Begräbnissstätten im Herzogthum Berg. - Mit zwei Zeichnungen von A. Bonnet. MonatsschrBergGV. 6, S. 233-236.

69. C. Rademacher. Germanische Begräbnissstätten am Niederrhein. Neueste Ausgrabungen 1898. - Nachrichten iber Deutsche Alterthumsfunde, $1899, \mathrm{~S} .29 \mathrm{ff}$.

70. F. Schmitz. Volksthümliches vom Siebengebirge. Fortsetzung. - RheinGBll. 4, S. 271-276; 334-345.

71. E. Pauls. Kulturgeschichtliches. Fortsetzung, 12. Zur Geschichte der Bienenzucht am Niederrhein. - ZBergGV. 34, s. $143-172$. 
Die historische Litteratur des Niederrheins für das Jahr 1899.153

72. O. Schell. Der ${ }_{7}$ Christbrand" im Bergisehen. - MonatsschrBergGV. 6, S. $247-249$.

73. Dronke. Die Eifel. Aus den nachgelassenen Papieren herausgegeben durch K. Küppers. Mit dem Bilde des Verfassers. Köln, Nenbner. VIII, $479 \mathrm{~S}$.

74. G. Virmond. Geschichte des Kreises Schleiden. - Schleiden, Braselmann VIII, $318 \mathrm{~S}$.

Dem Verfasser verdanken wir schon eine treffliche Geschichte der Eifeler Eisenindustrie, die ihren Hauptsitz in Kreise Schleiden hatte (vgl. Litteraturbericht 1897, nr. 79). Die vorliegende Arbeit ist hauptsachlich auf dem umfangreichen Sammelwerke Eiflia Illustrata von Schannat-Bärsch und der Geschichte des Dekanates Blankenheim von Becker (vgl. Litteraturbericht 1893, nr. 146) aufgebaut. Der erste allgemene Theil handelt über die Römer in der Eifel, die Eifel unter den Franken und die Einführung des Christenthums in der Eifel Der zweite spezielle Theil behandelt dann die Entstehung und die Geschichte der Eifeler Dyuastengeschlechter. Ein längerer Abschnitt ist auch der Geschichte der Primonstratenserabtei Steinfeld gewidmet. Dann folgt die Geschichte der einzelnen historisch wichtigeren Orte in alphabetischer Reihenfolge.

75. R. Kötzschke. Studien zur Verwaltungsgeschichte der Grossgrundherrschaft Werden an der Ruhr. Leipziger Habilitationsschrift. - Leipzig, Teubner. 52 S.

In einem der nächsten Hefte werden die Annalen eine ausführliche Besprechung bringen.

7วa). W. M. Becker. Die Initiative bei der Stiftung des Rheinischen Bundes. -- Giessener Dissertation. Giessen, Ricker IV, 86 S.

76. H. Forst. Die Spanier am Rhein im dreissigjährigen Kriege. - WZ. 18, S. 45-65.

Forst konmt zu dem Resultat, dass die Spanier keine glinzende Rolle gespielt haben, dem Kaiser nur geringen Nutzen gebracht, dagegen dem Kriege durch ihr Eingreifen von vorneherein einen internationalen Charakter verliehen hätten.

77. S. Riezler. Die Meuterei Johanns von Weerth. - Historische Zeitschrift 82, S. 38-97 und 192-239.

78. K. Nathan. Die Kämpfe zwischen Roer und Maas während des ersten Koalitionskrieges. - ZAachenGV. 21, S. 88 bis 121 .

Nathans Ausführungen, die sich nur auf Druckwerke stützen, 
geben vor allem eine Würdigung dieser Kämpte vom militärtechnischen Standpunkte aus.

79. P. Li e beskind. Geschichte des Füsilier-Regiments Fürst Karl Anton von Hohenzollern (Hohenzollernsches) nr 40. Mit 1 eingedruckten Skizze und 1 Bildniss. - Köln, Warnitz III, $64 \mathrm{~S}$.

80. J. Joesten. Litterarisches Leben am Rhein. Zwei Studien ïber: „Die litterarische Bildung am Rhein im vorigen Jahrhundert", "Gottfried Kinkel und sein Kreis in Bonn". Leipzig, Grunow. 127 S.

Im ersten Aufsatz sucht Joesten den Vorwurf der litterarischen Rückständigkeit der Rheinlande im 18. Jahrhundert zurückzuweisen. Im zweiten schildert er in liebender Anhanglichkeit den Entwicklungsgang Kinkels und die Geschichte des Maikäferbundes, einer Vereinigung von Freunden Kinkels in Bonn, in den $40 \mathrm{er}$ Jahren.

81. K. A. Bachofen von Echt. Beiträge zur Geschichte der Familie Bachoven von Echt. Zweite Auflage. - Wien, Selbstverlag. $45 \mathrm{~S}$.

82. E. Becker. Beiträge zur Geschichte der Familie Becker. Heft 1. Diisseldorf, Druck von Lintz.

\section{Ortsgeschichtliche Darstellungen.}

83. H. K e ussen. Beiträge zur Geschichte der Kölner Universität. - WZ. 18 , S. 315-369.

Die Arbeit zerfällt in zwei Theile. Der erste behandelt die ailteren Universitätsbibliotheken bis etwa 1500; die bedeutendste war die der Artistenfakultät, doch reichte sie nicht an die viel bedeutenderen Bibliotheken anderer Universitäten heran. Die Hauptmasse der Bibliotheken setzte sich zusammen aus Geschenken und Vermachtnissen verstorbener Protessoren. Ein eigenes Gebäude erhielt die Bibliothek trotz aller Bemühungen der Fakultat nicht. Im Anhang werden ältere Bücherverzeichnisse abgedruckt. Der zweite Theil giebt Nachrichten über den Kölner Aufenthalt des italienischen Humanisten Stephan Surigonus, die hauptsächlich aus dessen sonst recht unbedeutenden Gedichten geschöptt sind. Einige Proben seiner Dichtungen werden abgedruckt.

84. O. vou Bremen. Die Leprauntersuchungen der KöIner medizinischen Fakultät von 1495-1664. - WZ. 18, S. 65 bis 77 .

Der Aussatz begann sich um das Jahr 1000 in Mitteleuropa aus. zubreiten, wenn auch früher vereinzelte Fälle vorgekommen sein werden. 
Die historische Litteratur des Niederheins für das Jahr 1899. 155

Wie bei den alten Juden wandte man als Heilmittel die Isolierung an. Zur Unterbringung der Kranken hatte man in Köln ausser mehreren kleinen Hausern in Riehl, am Judenbüchel und in Rodenkirchen vor allen das grosse Leprosenhaus vor dem Aachener Thor. Die Untersuchuug der lepraveldächtigen Personen wurde ursprünglich von den i.eprosen selbst vorgenommen. Jedoch wurde, weil hierbei Missbräuche eingerissen waren, die Untersuchung später der medicinischen Fakultät uhretragen. Der erste derartige urkundlich belegte Fall fällt in das Jahr 1486: \%weifellos traben solche Untersuchungen aber schon lange vorher stattgetunden. Verfasser gewinnt ans den erhaltenen Untersuchungsprotoliollen den Eindruck, dass die Professoren ihres Antes mit grosser Gewissenhaftigkeit und augleich doch mit echter Menschentreundlichkeit gewaltet haben. Der Ruf der Kölner Untersuchungen muss bedeutend gewesen sein, da sich Patienten aus ganz West- und Nordwestdeutschland und aum den Niederlanden in Köln zur Untersuchung siellten.

8.5. S. Stedtfeld. Köln. Mïnzen. - JVARh. 104, S. 188-192. Ueber 2000 Münzen des 13. und 14. Jahrhunderts wurden bei der Anlage aer Rennbahn auf dem Weidenpescher Hof gefunden.

86. Spriichwörter und alte Volks- und Kinderlieder in Kölnischer Mundart. - Köln, Stauff $12^{\circ} .65 \mathrm{~S}$.

8i. R. Petseh. Kölnische Sprïchwörter und Kinderreime. Münchener AllgemeineZtg. Beilage $1899 \mathrm{nr} .123$.

86. L. Fränkel. Volkskundliches aus Johann Wilhelm Wolfs Kölner Jugenderinnerungen. — Zeitschr. für Volkskunde. 9. S. $351-361$.

Vgl. Litteraturber. 1898, nr. 226.

89. I. Schwörbel. Zur Geschichte des Kölner Stapelhauses. Stadtanzeiger der KölnZtg. 1899, nr. 224; 228; 241.

90. L. Schwörbel. Deutz zu Ende des 18. und zu Anfang des 19. Jahrhunderts. Stadtanz. der KölnZtg. 1899, nr. 354; 366; $378 ; 397 ; 469$.

91. H. Schafstaedt. Die Festung Mülheim am Rhein zu Ende des 16. Jahrhunderts und zu Anfang des 17. Jahrhunderts. Programm des Gymnasiums in Mülheim am Rhein. - Mülheim, Künstler $4^{0}$. $33 \mathrm{~S}$.

Schatstaedt giebt eine zuverlässige Geschichte von der krbauung der Festung Mülheim a. Rh. Auf Drängen der Nachbarstadt Köln, das sich bedroht fühlte und vielleicht auch eifersüchtig war, musste die Festung im Jahre 1615 wieder zerstört werden. Für die Darstellung sind die Archivalien des Kölner und Mülheimer Stadtarchivs fleissig benutzt worden. 
92. Aeg. Müller. Stade. - MonatsschrBergGV. 6, S. 98-101. Gut boi Bensberg, sehr triih im Besitz der Herrn von Loè.

93. A. Mïller. Eulenbroich. -- MonatsschrBergGr.6, S. 4-8. Gut bei Rösrath.

94. A. Müller. Forstbach. - MonatsschrBergGV. 6, S. 82 bis $8 \check{5}$.

95. Hans yon W indeck (J. Joesten). Alt-Bonn. - KölnTagebl. 1899, nr. 498; 500 .

96. H. Ingenhoven. Geschichte des Kreuzberges bei Bonn. [A. u. d. T. Bilder aus der Geschichte ron Bonn und seiner Umgebung. Heft 11.] - Bonn, Hauptmann. $38 \mathrm{~S}$.

Die Kirche auf dem Kreuzberge wurde im Jahre 1628 erbaut. Den Gottesdienst versahen bis 1802 die Serviten, von 1855 bis 1872 die Jesuiten und seit 1888 die Franziskaner.

97. C. Schultheis. Alte Befestigungen von Münstereifel. Mit 2 Tafeln. - JVARh. 104, S. 65-(06.

98. C. F ïssenich. Das Wappen von Berglueim an der Erft. RheinGBll. 4, S. $257-260$.

99. F. Schollen. Die rechtsgeschichtliche Bedeutung des Aachener Kurgerichts. - MVAachenVorzeit 11, S. 49-64.

100. E. Pauls. Beiträge zur neuern Geschichte Aachens. I. Zur Geschichte der Presse und der Censur in Aachen vor 1816. II. Zur Geschichte des Strassenkampfes in Aachen am 2. März 1793. III. Die Bemühungen der französischen Regierung um die Vermehrung der Rathliausbibliothek und um die Gründung eines städtischen Museums in den Jahren 1812 und 1813. - ZAachenGV. 21, S. 216-253.

101. H. Savelsberg. Zur Geschichte des Hauses nzum Horn in der Jakobstrasse. Mit Abbildungen. - MVAachen Vorzeit 12 , s. $31-45$.

102. E. Teichmann. Zur Namensgeschichte der Aachener St. Salvatorkapelle. - ZAachenGV. 21, S. 60-87.

Die Kapelle wird im Volksmunde Sent Sellester = St. Silvesterkapelle genannt. Teichmann nimmt an, dass der Name St. Salvator dem Volke unverständlich gewesen und dass daher der ihm durch die vielen Legenden ganz geläufige Name Silvester untergeschøben worden sei, was um so leichter war, als ein gewisser Gleichklang der Namen ja vorlag. 
Die historische Litteratur des Niederrheins für das Jahr 1899. 157

103. E. Pauls. Der Proserpinasarg in Aachen und die Sage von der Bestattung Karls des Grossen. - ZAachenGV. S. 259 bis 261 .

104. J. Fey. Zur Geschichte der Kirche von Marienberg. ZAachenGV. 21, S. 263-264.

105. C. Tucking. Die Entwicklung des Handels in Neuss. BGNenss-Grevenbroich. 1, S. 43-48; 57-64.

106. Johanniterkommende zu Neuss. - BGNeuss-Grevenbtoich. 1, S. $124-127$.

107. F[elten]. Ein Beitrag zur Geschichte der Neusser Kirmes. BGNeuss-Grevenbroich. 1, S. 93-95.

108. Einiges aus Heerdts Geschichte. - BGNeuss-Grevenbroich 1, S. $17-30 ; 33-42 ; 49-57 ; 65-71 ; 81-86$.

109. W. Haus Dyckhof und seine Besitzer. - BGNeuss-Grevenbroich 1 , S. $72 ; 87-91$.

Dyckhoff in der Gemeinde Büderich bei Neuss.

110. W. Weitere Nachrichten über den Dyckhof. Piperskapelle. BGNeuss-Grevenbroich. 1, S. 106-112.

111. F. W. Strauss. Beiträge zur Geschichte der Stadt MünchenGladbach und des Jülicher Landes. Neue Folge. MünchenGladbach, Stranss. $120 \mathrm{~s}$.

Compilation aus älteren Werken. Selbständige Forschungen sind ncht ang estellt worden.

112. $\mathrm{M}$ u s h a cke. Krefeld im fridericianischen Zeitalter, unter besonderer Berücksichtigung der Seidenindustrie. Vortraggehalten in der Krefelder Ortsgruppe des alldeutschen Verbandes. - Krefeld, Kramer \& Baum. $23 \mathrm{~S}$.

M. behandelt vor Allem die Verdienste der Mennonitenfamilie von dar Leyen um die Entwicklung der Seidenindustrie.

113. J. Real. Darstellungen Wachtendonks aus der Festungszeit. Veröffentlichungen des Historischen Vereins fiir Geldern und Umgegend. - Geldern, Muiller. $11 \mathrm{~S}$.

114. J. Real. Pläne und Ansichten der ehemaligen Festung Geldern. Veröffentlichungen des Historischen Vereins für Geldern und Umgegend. Geldern, Müller. $8 \mathrm{~S}$.

115. L. Henrichs. Die Mark Straelen und ihre zugehörigen Orte. Geldern, Schaffrath. 63 S. 
Mit gewohnter gründlicher Kenntniss und Benutzung des urkundlichen Materials stellt $H$. den L'mtang der alten Mark Straelen fest.

116. J. A s b a ch. Die Napoleonische Universität in Düsseldorf. Beilage zum Jahresbericht des Gymnasiums zu Düisseldorf. Düsseldorf, Voss. $4^{0} .32 \mathrm{~S}$.

Schon Murat hatte im Jahre 1806 die Errichtung einer Universit it in Düsseldorf für das Grossherzogthum Berg geplant, war jedoch an der Austihrung dureh den Krieg mit Preussen verhindest worden. Dureh ein von Napoleon am 17. Dez. 1811 erlasienes Dekret wurde sodaun die Errichtung einer Universität und eines Lyceums in Düsseldorf und einer grossen Anzahl Mittelschulen in den verschiedenen Städten des Grossherzogthums angeordnet. Die Universitat sollte fünt Fakultäten haben, die der Theologie, Jurisprudenz, Medizin, Mathematik und Naturwissenschaften und die der schonen Künste. Der kaiserliche Kommissar im Grossherzogthum Berg, Graf Beugnot. suchte die Sache auf jede Weise zu fördern. Der Ausgang des Feldzugs gegen Russland und dann Napoleons Niederlage bei Leipzig bereiteten dem Unternehmen ein rorzeitiges Ende.

11\%. A. We yersberg. Die in den privilegirten Handwerken der Solinger Industrie vertretenen Familiennamen. Zweiter Nachtrag. MonatsschrBergGV. 6, S. 23-25.

118. F. Jorde. Bilder aus dem alten Elberfeld. - Elberfeld, Baedeker. IV, $260 \mathrm{~S}$.

Die Bilder sind auf Grund archivalischen Materials und der neueren lokalhistorischen Forschungen entworfen. Die Darstellung ist so populär gehalten, dass sie stellenweise einzelne Zïge $z u$ frei erfundenen Erzählungen verarbeitet.

119. F. Jord e. Das Leichensingen im alten Elberfeld. - MonatsschrBergGV. 6, S. 150-15̄o.

120. W. Breidenbach. Statistisches aus Elberfeld. - MonatsschrBergGV. 6, S. 8-11.

Rentabilitätsberechnung der Elberfelder Müblen, aus der sich de Bevölkerungsziffern für die Jahre 1776-1791 ergeben.

121. J. V. Bredt. Barmen im 15. Jahrhundert. - MonatssehrBergGV. b, S. $113-130$.

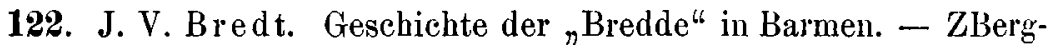
GV. 34, S. $123-132$.

Die Bredde war ein Hof in der Freiheit Barmen. Darnach ist die Familie Bredt benannt, deren Ahnherr, der 1660 verstorbene Johamn Bredt, früher den Namen Sibel geführt hatte.

122a. 0. Schell. Die ehemalige Brautkrone der reformirten Gemeinde zu Elberfeld. - MonatsschrBergGV. 6, S. 54-59. 


\section{Kirchengeschichte.}

123. Das Christenthum im römischen Köln. - RheinMerkur. 1899, nr. 171 und 177.

124. F[elten]. St. Quirinus, Stadtpatron. -- BGNeuss-Grevenbroich. 1, S. $90-106$.

125. F|elten]. Ein Deutsches Leben des hl. Marschalls Quirinus. BGNeuss-Grevenbroich. 1, S. 113-118.

126. Die Verehrung des hl. Quirinus. - BGNenss-Grevenbroich. 1, S. $129-137$.

127. H. Löbbel. Der Stifter des Karthäuserordens, der hl. Bruno aus Köln. - Münster, Schoningh $246 \mathrm{~S}$.

Verf. unter,ucht zunächst die für die Lebensbeschreibung in Betracht kommenden Quellen, wobei die Viten als werthlos ausscheiden. Auf Grund dieser Quellen giebt el dann eine Darstellung von Bruno's Lethen, wobei er das Feststehende von dem nur Wahrscheinlichen vorsichtiły scheidet. Der Heilige ist in Köln um das Jalı 1030 aus adligem Hause geboren. $O b$ er in Rheims, wo er seine theologische Bildung. erhielt. oder in Köln die Priesterweihe empfing, ist ungewiss. 1075 wurde er Kanzler den Erzb. Manasse von Rheims. Nachden er aber diesen der Simonie angeklagt hatte, ging er nach Kỏln, wo er Kanonikus an St Kunibert wurde. Nach Manasse's Absetzung wurde Bruno selber zum Erzbischof ron Rheims ernannt, gelangte jedoch nicht in den Besitz selnes Stuhles, worauf er sich dem Mönchleben zuwandte. Im Jahre 1090 wurde er von seinem Schüler Papst Urban II. nach Kom berufen; er bheb jerloch nicht lange hier, sondern gxündete in La Torre in Calabrien ene nene Carthause, in der er im Jahre 1101 starb. Bruno war auch schrif tellerisch als Exegut thätig. Früh schon wurde er als Heiliger verehrt, wenn auch eine eigentliche Kanonisation nicht stattgefunden hat.

128. F. X. F unck. Kirchengeschichtliche Abhandlungen und Untersuchungen. 2. Bd. nr. 21. Der Verfasser der Nachfolge Christi. -- Paderborn, Schöningh. V, 483 S.

Der Autsatz war früher im Historischen Jahrbuch der Göıresgesellschaft erschienen.

129. G. I b m. Thomas von Kempen und die Brüder vom gemeinsamen Leben. - Germania. Wissenschaftliche Beilage 1899 , nr. 14, 27, 49.

130. Arn. Steffens. Die Verlegung des Kollegiat-Kapitels von Stommeln nach Nideggen und von Nideggen nach Jülich. AnnHVNiederrh. 68. S. 109-132. 
131. H. Maassen. Geschichte der Pfarreien des Dekanates Bonn. II. Theil. Bonn Land. [A. u. d. T. Dumont, Geschichte der Pfarreien der Erzdiözese Köln. Nach den einzelnen Dekanaten geordnet. V. Dekanat Bonn. II. Theil.] - Bonn, Hanstein. X, $383 \mathrm{~S}$.

132. Th. H. Hürth und F. Hauptmann. Die Schutzpatrone von Bonn. [A. u. d. T. Bilder aus der Geschichte ron Bonu und seiner Umgebung 10.] - Bonn, Hauptmann. $68 \mathrm{~S}$.

Schutzpatrone sind die hl. Kassius, Florentius und Genossen, und seit 1702 die hl. Maria.

133. F. Hauptmann. Geschichte der Kevelaerbruderschaft. Zu ihrem 200 jährigen Jubiläum nach dem Archiv der Bruderschaft dargestellt. [A. u. d. T. Bilder aus der Umgebung von Bom und seiner Umgebung 12.] - Bonn, Hauptmann. $79 \mathrm{~s}$.

134. F[elten]. Zur Geschichte des Neusser Klarissenklosters. BGNeuss-Grevenbroich. 1, S. 91-93.

135. C. Tücking. Jakobsbrüder in Neuss. - BGNeuss-Grevenbroich. 1, S. 1-14.

Ursprünglich traten die Wallfahrer nach Santiago di Compostella von Fall zu Fall zusammen; später blieben sie nach der Vollendung ihrer Wallfahrt in Fühlung und bildeten die Jakobsbruderschaft.

136. E. D e m m er. Geschichte der Reformation am Niederrhein und der Entwicklung der evangelischen Kirche daselbst bis zum Jahre 1885. Mit Nachtrag. — Düsseldorf, Schaffnit. IX, 209 und $10 \mathrm{~s}$.

Neue Titelausgabe.

137. F. Kold ew e y. Johannes Monheim und die Kölner. Der erste Streit zwischen Jesuitismus und Protestantismus. - Zeitschrift für wissenschaftliche Theologie. 42, S. 106-138. Katechismus.

Betrifft den Streit wegen des 1560 erschienenen Monheim'schen

138. Bornefeld. Die Pfarrer der Gemeinde Lüttringhausen seit der Reformationszeit. - MonatsschrBergGV. 6, S. 34--39.

139. C. Clement. Zur Kirchengesehichte Düssels. - Monatsschr. BergGV. 6, S. 173-176. 
Die historische Litteratur des Niederrheins für das Jahr 1899. 161

\section{Kunstgeschichte.}

140. P. Clemen. Die Kunstdenkmäler des Kreises Bergheim. In Verbindung mit E. Polac\%ek bearbeitet. Mit 10 Tafeln und 82 Abbildungen im Text. [A. u. d. T. Die Kunstdenkmäler der Rheinprovinz. Im Auftrage des Provinzialverbandes herausgegeben. IV, 3.] - Diisseldorf, Schwann. VI, 168 S. Unter den kirchlichen Bauten ragen die Kirche zu Bergheim und die Stiftskirche zu Kerpen hervor. Dann findet sich eine Gruppe von Firchen, die in den Thürmen und ihren Achteckhelmen eine Verwandtschaft mit einander zeigen. Kunstgeschichtlich hervorragender sind dio Prołanbauten des Kreises, so vor Allem die Renaissanceschlösser zu Bedburg, Frens und Harff.

141. Frz. Büttgenbach. Die kirchliche Kunst in Monographien, Skizzen und Kunstbildern. Mit vielen Tafeln und Abbildungen im Texte. - Aachen, Schweitzer. $4^{0}$, X, 204 S.

142. Frz. B ï t $\mathrm{g}$ e $n \mathrm{~b}$ a $\mathrm{ch}$. Monographien iuber die kirchliche Kunst. Ergänzungen. Kirchenthüren und Altäre. Mit Abbildungen und 1 Tafel. - Aachen, Schweitzer. $4^{0}$. $51 \mathrm{~S}$.

143. F. Th. Helmken. Der Dom zu Köln, seine Gesehichte und Banweise, Bildwerke und Kunstschätze. Ein Führer für die Besucher. 4. Auflage. Mit Abbildungen. - Köln, Boisserée. VI, $164 \mathrm{~S}$.

144. M. Hasak. Die Kirche Gross St. Martin und St. Aposteln in Köln. Mit 11 Abbildungen und 7 Tafeln. [A. u. d. T. Die Baukunst. 1. Jahrgang, Heft 11.] - Berlin, Spemann. Folio. $16 \mathrm{~s}$.

145. 0. von Falke. Kölnische Hafnergeschire. Mit 1 Tafel. - Jahrbiicher der Königl. Prenssischen Kunstsammlungen, 19, S. $191-201$.

F. weist nach, dass in Köln in der ersten Hälfte des 16. Jahrhunderts eine blühende Thonindustrie bestanden hat. In der Maximinentrasse wurde vor einigen Jahren ein alter Thonofen aufgedeckt, wobei sich zahlreiche gut erhaltene Stücke fanden. Doch weist Falke auch eine Anzahl früher schon bekannter Stücke von hervorragendem Kunstwerthe, die man aber als Erzeugnisse der Nürnberger Thonindustrie ansprach, Köln zu. Gegen die Mitte des Jainrhunderts schritt der Rath gegen die Krug- und Kannenbäcker ein, um der Holzvertheuerung und der Feuersgefahr vorzubeugen.

Annalen des hist Vereins LXXII 
146. Greving. Die Bilder der Pfarrer von St. Columba. KBWZ. 18 , S. $32-40$.

147. E. Firmenich-Richart\%. Der Meister des hl. Bartholomäus. Studie zur Geschichte der altkölnischen Malerei. I. Mit Lichtdruck und zwei Abbildungen. - ZChrK. 12, S. $261-274$.

148. J. Marchand. Grabmäler in der St. Ursulakirche zu Köln. I. Das Grab der hl. Ursula. Mit 7 Abbildungen. - ZChrK. 12, S. 123-128. II. Das Grabmal der hl. Viventia. Mit 6 Abbildungen. - ZChrK. 12. S. 183-186.

149. A. Schnütgen. Die silbervergoldete hochgothische Monstranz des Kölner Domes. - ZChrK. 12, S. 225-230. Die Monstranz stammt aus dem Anfang des 15. Jahrhunderts:

150. A. Ditges. Emailblättchen des alten St. Kuniberts-Schreines im Museum zu Darmstadt. - ZChrK. 12, $\mathrm{s}$. 219-222.

151. O. Schell. Führer durch Altenberg im Dhünthal. Mit 2 Abbildungen. - Elberfeld, Baedeker. $55 \mathrm{~S}$.

152. H. Oidtmann. Nachrichten über Rheinisehe Glasmalereien des 16. Jahrhunderts insbesondere über die Glasgemälde in der Burgkapelle za Ehreshofen, ein spätes Werk monumentaler Glasmalerei in den Rheinlanden. Mit Abbildungen. ZChrK. 12. S. 55-64;67-74.

153. W. Ef f mann. Die karolingisch-ottonischen Bauten zu Werden. I. Stephanskirche, Salvatorskirche, Peterskirche. Mit 288 Textfiguren und 21 Tafeln. - Strassburg, Heitz. XI, $447 \mathrm{~S}$.

Effmann unterzieht die alten Kirchen Werdens einer eingehenden baugeschichtlichen Untersuchung. Der vorliegende erste Band behandelt die aus karolingischer Zeit stammenden Kirchen; diese liegen sämmtlich unmittelbar bei dem Kloster. Sogleich nach Errichtung des Klosters im Jahre 799 begann der hl. Ludger mit dem Bau einer Kirche, der Stephanskirche, die auch einige Jahre später vollendet und eingeweiht wurde. Im 16. Jahrhundert ist die Kirche umgebaut und im Jahre 1760 abgebrochen worden. Die Kirche ist nach den von der altchristlichen Kunst gegebenen Vorbildern errichtet worden und stellt sich in ihrer Dreiconchenform als eine direkte Uebertragung aus Italien dar, wo der hl. Ludicer diese Form an den Memorienkirchen selbst kennen gelernt hatte. Wenige Jahre nach der Vollendung der Stephanskirche wurde wahrscheinlich noch von Ludger selber der Bau einer grösseren Kloster- 
Die h1storische Litteratur des Niederrheins für das Jahr 1899. 163

kurche, der Salvatorskirche, begonnen. Der ursprüngliche Plan wurde wohl bald nach Ludgers Tod geändert, inden die Kirche durch die Hnzufügung der Ludgerus- und Ludgeridenkrypta - der Grabstätten tur dell hl. Ludger und seine Anverwandten, die Ludgeriden - nach osten verlangert wurde. Die Krypten, die sich gleichfalls altchristlichen bautormen anschlossen, wurden um 830 vollendet. Die Ludgeruskrypta ist noch in ihrer ursprünglichen Form erhalten, während die Ludgeridenkivpta unter Abt Gero im Jahre 1059 umgebaut worden ist; doch lasst skcl der ursprüngliche Grundriss noch nachweisen. Die Salvatorskirche wurde in Jahre 875 eingeweiht. Sie war eine querschifflose, dreischiffige Basilika mit gewölbten Seitenschiffen. Durch Brande und den didurch veranlassten Neubau des 13. Jahrhunderts wurde diese Kirche bis auf wenige noch aufrechtstehende Reste (ausser der Ludgeruslsrypta) zer'stört. Doch lässt sich der Grundriss durch die unter dem Fussboden noch erhaltenen Fundamentmauern rekonstruiren. Sofort nach ihrer Vollendung solite die Salvatorskirche auch mit einer nach Westen sich anschliessenden Kirche, der Peterskirche, ausgestattct werden, die aber er-t 943 eingreweiht werden konnte. Die Peterskirche sollte dem Sendgercht für seine Sitzungen und auch pfarramtlichen Zwecken dienen und so die Klosterkirche entlasten. Diese Kirche ist weder ein Rest, noch eine Nachbildung der 875 eingeweihten Salvatorskirche, sondern ein selbständiges als Centralbau gedachtes Gebäude. Die Baugeschichte diever verschiedenen Baulichkeiten wird bis in die neueste Zeit hinein verfolgt. Die Ergebnisse der mit grosser Sorgfalt ausgeführten Untersuchungen, die vielfach von den bisherigen Ansichten abweichen, dürtten sicher stehen und sind von grosser Bedeutung für die allgemeine Baugeschichte.

154. H. Schrörs. Zur Baugeschichte der Kirche auf dem Apollinarisberge. - AnuHVNiederrh. 67, S. 130-136.

15̄. J. B u chkremer. Der Königsstuhl der Aachener Pfalzkapelle und seine Umgebung. Mit einer Tafel und einer Abbildung. - ZAachenGV. 21, S. 135-194.

Bei den Wiederherstellungsarbeiten im Aachener Münster ist in neuerer Zeit auch der Königsstuhl in Betracht gekommen; dieser stammt aus karolingischer Zeit und ist eines der ältesten und ehrwürdigsten l)enkmäler des Münsters. Buchkremer giebt eine genaue Beschreibung: des ursprünglichen Zustandes des Krbnigsstuhles und seiner Umgebung, der mit der Zeit vorgenommenen Aenderungen und der jetzt beendeten Wiederherstellung.

156. A. Richel. Die Denkmünzen auf den Aachener Frieden von 1748. Mit 5 Tafeln. - ZAachenGV. 21, S. 195 bis 215 .

15\%. P. Redlich. Unterhandlungen über den Verkauf des Rubens- 
schen Gemäldes in der Kapuzinerkirche zu Aachen. ZAachenGV. 21, S. 254-258.

158. Potthast. Die Stiftskirche zu Germersheim. - MonatsschrBergGV. 6, S. 101-106

159. W. Sauer. Ueber Siegel der Grafen Adolf III., Adolf IV., Dietrich II. und Gerhard von der Mark. - ZBergGV. 34, S. $267-275$.

160. Richter. Zwei interessante Grabsteine der Grafen von Broich. - MonatsschrBergGV. 6. S. 52-54.

Die Grabsteine befinden sich in der evangelischen Kirche zu Marienthal (Rheinpfalz).

161. H. Höfer. Beiträge zur Geschichte der Kunst und der Kunstbestrebungen der Cisterzienser in deu Rheinlanden von der Stiftung des Ordens bis zur Aufhebung. - Studien und Mittheilungen aus dem Benedictiner- und Cistercienser-Orden. 20 , S. 3-25; 335-347; 615-627.

Behandelt die Klöster Altenberg, Camp und Heisterhach.

\section{Biographien und Aehnliches.}

162. F. W. E. Roth. Adam Volemar zu Köln in seinen Beziehungen zu Nicolaus Wollick und Heinrich Glareanus 1501 bis 1510. - AnnHVNiederrh. 68. S. 190-193.

163. F[elten]. Das Leben des Tertiariers Heinrich von der Blume. - BGNeuss-Grevenbroich. I, S. 119-124.

164. E. Johann Kaspar Kratz. Ein rheinischer Glaubenszeuge. KölnVolksZtg. 1899, nr. 307.

Geboren zu Golzheim bei Düren, hingerichtet in Tonkin im Jahre 1737, Januur 12.

165. H. Schnock. Zur Geschichte Mare Antoine Berdolets, des ersten und einzigen Bischofs von Aachen. -. MVAachenVorzeit. 12 , S. $1-31$.

166. P. Höveler. Kardinal Krementz, Generalvikar Kleinheidt, Domkapellmeister Koenen und Professor Scheeben. Vier Charakterbilder aus der jüngsten kölnischen Kirchengeschichte. Mit 4 Porträts. - Diusseldorf, Schwann. 64 S.

167. Weihbisehof Hermann Josef Schmitz. - KölnVolksZtg. 1899, nr. 953. 
Die historische Litteratur des Niederrheing für das Jahr 1899. 165

168. Bischof Dr. Hubertus Simar, Der erwählte Erzbischof von Köln. Ein Lebenstbild. Mit Titelbild und 6 Abbildungen. Köln, Bachem. $12^{0} .64 \mathrm{~S}$.

169. Hubertus Simar, Erzbischof von Köln. Ein Lebensbild, den Katholiken der Erzdiözese gewidmet. Mit Porträt und Wappen. - Essen, Fredebeul \& Koenen. $12^{\circ} .32 \mathrm{~S}$.

170. P. Höveler. Adolf Kolping als katholischer Volksschriftsteller. - Diisseldorf, Schwann. $48 \mathrm{~S}$.

171. Hans von Windeck. Göthe in Bonn. Ein Blatt der Erinnerung. - KölnTagebl. $1899 \mathrm{nr}$. 523.

179. G. Karpeles. Heinrich Heine. Aus seinem Leben und aus seiner Zeit. - Leipzig, Titze IV, $347 \mathrm{~S}$.

173. J. A s b a c h. Heine und das Düsseldorfer Lyceum. Münchener Allgemeine Zeitung. Beilage 1899 nr. 246, $257,279$.

174. H. Hiiffer. Zu Heines Geburstagsfeier. - Dentsche Rundschau. 101, S. $498 \mathrm{f}$.

175. J. Andries. Der rheinisch-westfälische Dichter Enil Rittershaus. Sein Leben und Wirken. Programm des Gymnasiums an St. Aposteln in Köln. - Köln, Druck von Bachem. $4^{\circ}$. $16 \mathrm{~S}$.

176. L. Pastor. Angust Reichensperger 1808-1895. Sein Leben und sein Wirken auf dem Gebiete der Politik, der Kunst und der Wissenschaft. Mit Benutzung seines ungedruckten Nachlasses dargestellt. - Freiburg, Herder. Bd. 1. Mit einer Heliogravüre und einem Lichtdruck. XXV, $606 \mathrm{~S}$. Bd. 2. Mit zwei Lichtdrucken. XV, $496 \mathrm{~S}$.

Ein reiches Material stand Pastor für seine Darstellung zur Verlugung. Zunächst das Tagebuch, das Reichensperger von seiner Jugend all gefuhrt hat, dann ein sehr umfangreicher Briefwechsel, seine Schriften und Aufsätze und seine Reden in Pariamenten und Versammlungen. Das Leben R.s. zerfällt in zwei grosse Theile: 1. Die Lehr- und WanderJahre von 1808 bis c. 1840; 2. Die Jahre der Wirksamkeit und des Kamptes. In das Ende der ersten Periode fällt ein Ereigniss, das für Reichenspergers Entwicklung bestimmend war, nämlich die Verhaftung und Abführung des Kölner Erzbischofs und damit der erste Konflikt des Preussischen Staates mit der katholischen Kirche. Es brachte ihm zum Bewusstsein, dass er katholisch sei, während sich bei ihm früher cine gewisse Gleichgültigkeit gegen die Kirche fand. Mit den $40 \mathrm{er}$ 
166 Kaspar Keller: Die historische Litteratur des Niederrheins etc.

Jahren begannen für R. die Jahıe des Wirkens auf kunstgeschichtlichem und politischen Gebiet. Auf jenem galt sein Streben der Vollendung des Kölner Domes und der Wiederbelebung der gothischen Kunst. Für sein politisches Leben waren die Kolner Wirren der Ausgangspunkt. Pastor schildert ausführlich den Entwicklungsgang Reichenspergers als Politiker, bei dem auch die naturgemässe Reaktion des Rheinländers und des rheinischen Wesens gegen das ostelbische Wesen mitbestimmend wirkte, seine Stellung in der 1848 er Bewegung, seine Thätigkeit als Abgeordneter in Frankfurt und Erfurt, in der Landrathskammer und zur Konfliktszeit, und endlich zur Zeit des Kulturkampfes. Die letzten 10 Jahre hatte sich R. vom politischen Leben ganz zurückgezogen.

17\%. R. E. Hackenberg. Der rothe Becker. Ein deutsches Lebensbild des 19. Jahrhunderts. Mit Porträt. - Leipzig, Baedeker. VII, 316 s. 